\section{Apnéia do Sono em Obesos}

\section{RESUMO}

Obesidade tem alta prevalência na população geral gerando custos econômicos para a sociedade. Individuos obesos apresentam risco maior de desenvolver de diabetes mellitus, doenças cardiovasculares, dislipidemia, e outras doenças crônicas como a Síndrome da Apnéia Obstrutiva do Sono (SAOS). A SAOS é uma doença crônica, progressiva, incapacitante, com alta mortalidade e morbidade cardiovascular. Os sintomas noturnos incluem roncos, pausas respiratórias, sono agitado com múltiplos despertares, noctúria e sudorese. Os sintomas diurnos são principalmente sonolência excessiva, cefaléia matinal, déficits neurocognitivos, alteraçōes de personalidade, redução da libido, sintomas depressivos e ansiedade. Fatores anatômicos e fatores funcionais contribuem para esta instabilidade das VAS. O diagnóstico de confirmação é feito pela polissonografia, que também estabelece critérios de gravidade. O tratamento está centrado em quatro pontos: tratamento da obesidade, tratamento comportamental da SAOS, tratamento físico e procedimentos cirúrgicos. Em pacientes selecionados algumas drogas podem ser úteis no sentido de diminuir o número ou a duração dos períodos de apnéia. A possibilidade de hipotiroidismo deve sempre ser descartada, uma vez que em alguns casos a reposição com tiroxina pode levar a desaparecimento da apnéia. Também foram desenvolvidos aparelhos intra-orais removiveis e pacientes menos obesos e com alteraçōes específicas palatofaríngeas apresentam melhores chances de resposta cirúrgica à úvulo-pálato-faringoplastia. (Arq Bras Endocrinol Metab 2000; 44/1: 81-90)

Unitermos: Apnéia do sono, Obesidade, Diagnóstico, Tratamento

\begin{abstract}
Obesity is a disease with high prevalence and obese individuals have a higher risk of cardiovascular diseases, diabetes mellitus, dyslipidemia and Obstructive Sleep Apnea Syndrome (OSAS). OSAS is a chronic, progressive, incapacitating disease with high cardiovascular mortality and morbidity. Clinical symptoms include snoring, respiratory pauses, agitated sleep, nocturia, sudoresis, excessive sleepiness, headache in the morning, neurocognitive deficits, personality and libido changes, depressive symptoms and irritability. Anatomic and functional factors can contribute to the instability of the upper respiratory tract. The confirmations is made by the polisomnography, that also stablish severity criteria. Treatment is based in four points: obesity, behavioral, physical and surgical treatments. In selected patients, some drugs can be useful. Hypothyroidism must be always screened. Intraoral ortesis were developed and patients with normal weight with specific palatopharingeal changes have better chances to success in surgery (uvulopalatopharingoplasty)
\end{abstract} (Arq Bras Endocrinol Metab 2000; 44/1: 81-90)

Keywords: Sleep apnea; Obesity; Diagnosis; Treatment

\section{artigo original}

\author{
Marcio C. Mancini \\ Flavio Aloe \\ Stella Tavares
}

\begin{abstract}
Serviço de Clinica Médica de Emergência e Grupo de Obesidade e Doenças Metabólicas do Serviço de Endocrinologia e Metabologia do Hospital das Clinicas da Faculdade de Medicina da Universidade de Säo Paulo, Centro Interdepartamental para Estudos do Sono (CIES) do Hospital das Clinicas da Faculdade de Medicina da Universidade de São Paulo e Centro de Distúrbios do Sono do Hospital Israelita Albert Einstein, São Paulo, SP.
\end{abstract}


$\mathbf{O}^{\text {s }}$ BESIDADE É UMA DOENÇA de alta prevalência na população geral gerando custos econômicos elevados para a sociedade. Indivíduos acima do peso apre sentam riscos de desenvolvimento de diabetes mellitus, doenças cardiovasculares, dislipidemia, e outras doenças crônicas como a Síndrome da Apnéia Obstrutiva do Sono (SAOS), quando comparados com pessoas de peso normal. Indivíduos obesos mórbidos têm esses riscos ainda mais acentuados, com mais impacto sobre os índices de mortalidade.

A SAOS é uma condição médica importante por causa de sua morbidade e mortalidade. Como acomete preferencialmente indivíduos obesos, é fundamental que o endocrinologista tenha conhecimento da fisiopatologia, sintomas e tratamento da SAOS.

\section{DEFINIÇÃO}

As pausas respiratórias durante o sono (apnéias ou hipopnéias) são definidas como uma parada (apnéia) ou redução (hipopnéia) da passagem de ar pelas vias aéreas superiores (VAS), com duração mínima de 10 segundos. Estes eventos respiratórios ocorrem inúmeras vezes (até centenas de vezes) e, exclusivamente durante o sono, determinando sintomas c sinais que caracterizam a SAOS.

A SAOS é uma doença crônica, progressiva, incapacitante, com alta mortalidade c morbidade cardiovascular. A prevalência de SAOS é de 9\% da população masculina de meia idade (30-60 anos) e 4\% da população feminina após a menopausa. A prevalência de SAOS é ainda maior em faixas etárias avançadas. A SAOS pode ocorrer em qualquer idade, mas acomete principalmente homens obesos entre 40 e 60 anos, sendo menos freqüente em mulheres antes da menopausa. Em obesos mórbidos a incidência ultrapassa $50 \%$. O sexo masculino é mais afetado (proporção de 1,4 a 3,6 homens/mulheres) devido a diferenças anatômicas das VAS, perfil hormonal e distribuição adiposa do tipo central nos homens (tronco e pescoço). Os sintomas da SAOS podem ser classificados em noturnos e diurnos.

\section{Sintomas Noturnos}

Os sintomas noturnos incluem roncos, pausas respiratórias, sono agitado com múltiplos despertares, noctúria e sudorese.

\section{Roncos}

Ronco é um ruído produzido involuntariamente durante o sono pela vibração de alta freqüência da úvula, pálato mole e paredes faríngeas, epiglote e língua. Roncos estão
Tabela 1. Escala de graduação do ronco.

\begin{tabular}{ll}
\hline zero pontos: & sem roncos \\
l a 3 pontos: & presença de roncos, não interrompe o sono \\
& do parceiro (a). \\
4 a 6 pontos: & incomoda o parceiro (a) \\
7 a 9 pontos: & incomoda muito o parceiro (a) que não sai \\
10 pontos: & $\begin{array}{l}\text { incombiente de dormir } \\
\text { ambiente de dormir }\end{array}$ \\
\hline
\end{tabular}

presentes em cerca de 90 a $95 \%$ dos casos de SAOS. No início, é comum o cônjuge do roncador referir que o ronco era contínuo sem interrupçoos. Com a progressão da SAOS, as pausas respiratórias aparecem e o ronco passa a ser descontínuo e de maior intensidade. Para se avaliar a intensidade e impacto do ronco, pode-se utilizar a escala de graduação de ronco (tabela 1). Atualmente, sabe-se que a presença de ronco está associada a morbidade e mortalidade vascular em pacientes de meia idade, isto ć, hipertensão arterial, angina, doença isquêmica do miocárdio e AVCs, independentemente de outros fatores de riseo.

\section{Noctúria}

A noctúria ocorre por aumento da secreção de fator natriurético atrial.

\section{Sintomas Diurnos}

Os sintomas diurnos são principalmente sonolência excessiva, cefalćia matinal, déficits neuro-cognitivos, alteraçōes de personalidade, redução da libido, sintomas depressivos, ansiedade.

\section{Sonolência excessiva}

Sonolência excessiva é um sintoma complexo composto pelas experiências subjetivas que os pacientes descrevem e pelas medidas objetivas mensuráveis e comparáveis entre si. Sonolência excessiva pode ser definida clinicamente como dificuldade para manter um nível de alerta desejado ou como uma quantidade excessiva de sono. $\mathrm{O}$ paciente pode se queixar de cansaço, falta de energia, dificuldade de concentração, atenção ou indisposição ao invés de usar o termo sonolência. Sonolência pode se apresentar também como uma dificuldade para sustentar atenção e vigília em tarefas monótonas. O médico deve pesquisar se o paciente apresenta cochilos inadvertidos em situações sedentárias. Para avaliação subjetiva de sonolência diurna pode-se empregar escalas como a Epworth Sleepiness Scale. Trata-se de um questionário de 8 itcns graduado de 0 a 3 pontos para cada item, voltado para a avaliação subjetiva do grau de sonolência (Tabela 2). A avaliação objetiva da sonolência ć feita pelo 
Tabela 2. Escala de Sonolência Epworth.

Qual é a probabilidade de você "cochilar" ou adormecer nas situações que serão apresentadas a seguir, em contraste com estar sentindo-se simplesmente cansado? Isto diz respeito ao seu modo de vida comum, nos tempos atuais. Ainda que você não tenha feito, ou passado por nenhuma destas situações, tente calcular como poderiam tê-lo afetado. Utilize a escala apresentada a seguir para escolher o número mais apropriado para cada situaçáo.

$0=$ nenhuma chance de cochilar

$2=$ moderada chance de cochilar

SITUAĊ̃̃O

Sentado e lendo:

Vendo TV:

Sentado em um lugar público (ex.: sala de espera, igreja):

Como passageiro de trem, carro ou ônibus, andando uma hora sem parar:

Deitando-se para descansar à tarde, quando as circunstâncias permitem:

Sentado e conversando com alguém:

Sentado calmamente após almoço sem álcool:

Se você estiver dirigindo, enquanto pára por alguns minutos em trânsito intenso:

TOTAL:

\section{1 = pequena chance de cochilar \\ $3=$ alta chance de cochilar}

CHANCE DE COCHILAR

Johns MW. A new method for measuring daytime sleepiness: The Epworth Sleepiness Scale. Sleep 1991:14:540-5.

teste de latências múltiplas no laboratório de sono. Este é o procedimento padrão mais preciso para a quantificação objetiva de sonolência diurna. Consiste em 4 a 5 registros poligráficos durante o dia, realizados em intervalos de 2 horas, com duração de 20 minutos. Em cada registro, mede-se o tempo que o paciente demora para adormecer, calculando-se então a latência média de sono, sendo que um valor inferior a 5 minutos é considerado anormal. A sonolência excessiva nos pacientes com SAOS é provocada pelo número de despertares breves durante o sono noturno. Estes despertares são temporalmente relacionados com o término das pausas respiratórias. Os despertares breves não são lembrados pelo paciente e causam intensa fragmentação do sono sem reduzir necessariamente o tempo total de sono.

\section{Cefaléias}

Dores de cabeça em peso, bilateral, frontal ou occipital ou nucal ao despertar de manhã são comuns em pacientes com SAOS. As pausas respiratórias durante o sono podem causar hipercapnia, especialmente em pacientes com SAOS grave, e a vasodilatação reflexa desencadeada pelo acúmulo de $\mathrm{CO}_{2}$ causa dores que geralmente desaparecem em algumas horas após o despertar. Esta dor de cabeça é mais intensa quanto mais o paciente permanece dormindo por causa do maior acúmulo de $\mathrm{CO}_{2}$. A ativação simpática com aumento da pressão arterial sistêmica durante o sono pode causar cefaléias occipitais ou nucais.

\section{Sintomas neuropsicológicos e psiquiátricos}

Déficits cognitivos são comuns nos casos de SAOS, desde leve à grave, e podem ser comprovados através de testes neuropsicológicos específicos. Déficits de memória e atenção são causados pela sonolência excessiva e revertem com o tratamento. A fragmentação experimental do sono NREM e REM em indivíduos normais demonstra uma relação entre o número de despertares e déficits de memória e atenção. O sono REM está associado com a ncuro transmissão acetilcolinérgica, processamento de memória, sonhos, e a fragmentação do sono REM contribui com a disfunção de memória. Os déficits de funções psicomotoras mais complexas descritas acima são causados pela hipoxemia sobre o lobo frontal e persistem após o tratamento. A sonolência excessiva e os roncos altos causam desajustes conjugais, familiares e profissionais, não sendo incomuns relatos de separações de casais ou de declínio profissional, e sintomas depressivos. O risco de acidentes automobilísticos por causa da sonolência é 2 a 7 vezes maior em pacientes com SAOS do que na populaçào normal.

\section{Fisiopatologia da oclusão das vias aéreas superiores na SAOS}

A característica principal funcional das VAS dos pacientes com roncos primários e dos pacientes com SAOS é uma instabilidade que leva ao colapso exclusivamente durante o sono. Este colapso das VAS é o produto final da interação entre fatores anatômicos e funcionais, que causam um desequilíbrio entre a pressão de sucção inspiratória intrafaríngea e as forças dilatadoras dos músculos faríngeos das VAS. A base da língua e o pálato mole colabam a oro e hipofaringe, interrompendo a passagem do ar. Uma vez ocorrido o colapso das VAS com ausência de fluxo de ar, a satu- 
ração de oxihemoglobina diminui, ocorrendo um despertar com reabertura das vias respiratórias. Fatores anatômicos e fatores funcionais contribuem para esta instabilidade das VAS.

\section{Fatores Anatômicos}

As anormalidades anatômicas com estreitamento ocorrem em todos os níveis das VAS. Alterações macroscópicas como micrognatia, retrognatia, hipertrofia tonsilar e adenoidal, macroglossia ou depósito de gordura ou alterações microscópicas na mucosa por causa do trauma mecânico do ronco. A obesidade é um fator importante na fisiopatologia da SAOS por causar modificações de tamanho e formato nas VAS. Contudo, a maioria dos pacientes apresentam alterações anatômicas que só podem ser demonstradas com técnicas especializadas. Estas técnicas são, por exemplo, ressonância nuclear magnética (RNM), tomografia computadorizada, reflexão acústica e espectroscopia de prótons ou cefalometria. Estas técnicas comprovam que o tamanho das VAS durante o sono e em vigília é menor nos pacientes com SAOS do que roncadores e pessoas normais. A geometria das VAS está alterada na SAOS. Cortes transversais na ressonância magnética (RNM) de orofaringe mostram alterações onde o eixo maior situa-se no sentido ântero-posterior, ao contrário dos normais, cujo eixo maior situa-se no sentido látero-lateral.

\section{Alterações funcionais}

A disfunção neuro-muscular das VAS durante o sono pode ter uma causa específica mais evidente, como, por exemplo, em doenças como pontomielite, Síndrome de Shy Drager, Arnold-Chiari, de Parkinson, degeneração olivo-ponto-cerebelar, acidente vascularcerebral, seringomielia e esclerose múltipla. As VAS de pacientes com SAOS se comportam de maneira diferente do que em indivíduos normais. O grau de redução do tamanho (volume e área) das VAS, quando submetidas à uma pressão negativa, é significativamente maior $\mathrm{em}$ pacientes com SAOS. A pressão crítica ( $P$ crit valor numérico da pressão negativa quando as VAS colabam) de fechamento das VAS é positiva em pacientes com SAOS quando deveria ser negativa como $\mathrm{em}$ pessoas normais. Existem evidências mostrando que a atividade e o controle da musculatura dilatadora das VAS na SAOS são anormais. O estreitamento anatômico das VAS (obesidade ou outro fator anatômico) é relacionado com uma hiperatividade tônica neuromuscular para manter as VAS abertas. Portanto, o nível basal de ativação neuro-muscular tônica da faringe é substancialmente mais alto no paciente para compensar a redução do calibre das VAS. Durante o sono, este fenômeno compensatório neuromuscular se reduz, permitindo o colabamento transitório das VAS. Os episódios obstrutivos são caracterizados por perda transitória desta hiperatividade muscular, e a obstrução é terminada com uma intensa atividade fásica da musculatura dilatadora faríngea acima do basal. Isto pode ocorrer centenas de vezes durante a noite. A hipóxia e hipercapnia durante as apnéias causam uma depressão da atividade neuromuscular do genioglosso, pálatoglosso, tensor do véu palatino, piorando mais ainda a disfunção das VAS. A disfunção neuromuscular das VAS também se acentua com fragmentação do sono. Em cada episódio obstrutivo, a miofibrila da musculatura faríngea é ativada fasicamente de uma maneira intensa e acima do nível basal quando se encontra relaxada e em seu comprimento máximo. No início a contração é isométrica porque a VAS está colabada e fixa por causa da pressão negativa gerada pela musculatura do tórax e diafragma e, ao vencer a resistência, a contração passa a ser isotônica. Devido a estas circunstâncias, isto é, aumento do tônus muscular em vigília, regime de hipóxia e hipercapnia, trauma mecânico crônico e contração isométrica intensa, a musculatura faríngea sofre alterações progressivas de natureza bioquímica e histológica com aumento do número de miofibrilas de contração rápida e de miofibrilas morfologicamente anormais, que interferem na função muscular causando uma deterioração progressiva da função neuromuscular. A mucosa da VAS possui receptores de mucosa sensíveis a alterações de pressão. Durante um estímulo com pressão negativa, a musculatura dilatadora das VAS responde com aumento da atividade musculatura dilatadora. Tratase, portanto, de um arco reflexo local bulbar e rápido. O tempo de latência deste arco reflexo está aumentado em pacientes com SAOS, a magnitude da resposta muscular está reduzida durante o sono, apesar de a pressão negativa desenvolvida durante a inspiração em pacientes com SAOS, pode chegar até $-40 \mathrm{~cm}$ de água $(\mathrm{em}$ normais $=$ entre -2 e $-4 \mathrm{~cm}$ de água $)$. Edema, processo inflamatório crônico, redução da sensibilidade por lesão mecânica de receptores podem se as causas destas alterações deste reflexo.

\section{Alterações autonômicas na SAOS}

\section{Alterações agudas}

Durante as pausas respiratórias, a hipóxia e a hipercapnia estimulam os corpos carotídeos e os quimiorreceptores centrais que ativam de forma fásica o sistema reticular ascendente do tronco cerebral. Ocorre tam- 
bém a ativação fásica do SNA simpático e centros respiratórios, aumento do esforço respiratório até ocorrer um despertar com reabertura das VAS e retorno da respiração com hiperventilação reflexa. Estas alterações gasimétricas, o esforço respiratório contra a glote fechada (manobra de Müller) e a reação de despertar desencadeiam uma hiperatividade simpática aguda máxima ao final de cada pausa respiratória, podendo causar arritmias cardíacas (bradicardia-taquicardia, bloqueios átrio-ventriculares, extrassístoles, assistolias, etc.), variações significativas do débito cardíaco, da pressão arterial sistêmica e pulmonar, da pressão de perfusão cerebral. Estes fenômenos ocorrem a cada pausa respiratória.

\section{Alterações crônicas}

A hiperativação aguda e repetitiva do SNA, relacionada com as pausas respiratórias, causa um aumento tônico da atividade do SNA com alteração da sensibilidade dos quimiorreceptores e barorreceptores. Este aumento do tônus durante o sono aparece também durante a vigília, em função da gravidade e do tempo de evolução, e determinando o aparecimento de hipertensão arterial sistêmica (HAS). Este aumento tônico da atividade do SNA durante o sono e vigília em pacientes com SAOS causa a manutenção da PA durante a noite, enquanto que em pessoas normais a curva de 24 horas da PA apresenta uma queda durante o sono. Dosagem de catecolaminas, cortisol no sangue periférico e medidas da atividade neuro-muscular simpática (medida pela eletroneurografia de nervo sural) comprovam o aumento do tônus simpático e do eixo hipotálamo-hipófise em pacientes com SAOS. Estas medidas classicamente se normalizam com a traqueostomia ou tratamento com CPAP. A hipertensão arterial sistêmica está relacionada ao aumento do tônus simpático documentado pela dosagem de catecolaminas. Existe uma forte associação entre SAOS e hipertensão arterial sistêmica (HAS). Cerca de 40-81\% dos pacientes com SAOS apresentam HAS independentemente de peso, idade e sexo e $26-48 \%$ dos hipertensos apresentam SAOS. Ocorre hipertensão pulmonar, hipertrofia de ventrículo direito e esquerdo, cor pulmonale, insuficiência cardíaca direita e edema de membros inferiores. Entre as diversas alterações neuroendócrinas, há o desenvolvimento de resistência a insulina, intolerância a glicose, redução da atividade do sistema renina-angiotensina-aldostcrona, aumento da secreção de fator natriurético atrial e redução da secreção do hormônio de crescimento $(\mathrm{GH})$. A resistência à insulina e a redução do hormônio do crescimento (que estimula a lipólise) estão rela- cionadas com a obesidade de tronco na SAOS. Diversos estudos populacionais demonstram aumento da mortalidade na SAOS por infarto agudo do miocárdio (IAM), acidente vascular cerebral isquêmico (AVCI), acidente vascular cercbral hemorrágico $(\mathrm{AVCH})$ ou morte súbita. Cerca de $16 \%$ dos pacientes infartados apresentam SAOS. A causa mais freqüente de morte na SAOS é o IAM. Aumento da agregação plaquetária e redução da atividade fibrinolítica são fatores que contribuem para a ocorrência de IAM e AVCIs na SAOS.

\section{Despertares e Fragmentação do Sono}

A fragmentação do sono e os despertares ocupam uma posição central na determinação de alterações clínicas e laboratoriais da SAOS. Os despertares causam fragmentação do sono, sonolência diurna, déficits cognitivos e contribuem para as alterações cardiovasculares com ativação do SNA. Preparações experimentais em voluntários normais submetidos a despertares provocados por sinal sonoro sem alterações gasimétricas causam intenso aumento, mas transitório, da PA, FC, ventilação, elevação fásica do tônus do sistema nervoso autonômico simpático. Isto demonstra que os despertares sem alterações gasimétricas contribuem para as alterações cárdio-respiratórias agudas e crônicas na SAOS. Mesmo despertares mínimos produzem aumento do tônus simpático com elevação robusta da pressão arterial e freqüência cardíaca. $O$ despertar desencadeia retomada da ventilação porque o tônus muscular da VAS retorna ao nível de vigília, desobstruindo as VAS. Hipoxemia e hipercapnia contribuem para o desencadeamento do despertar, ocorrendo simultaneamente com esforço respiratório máximo ao final das pausas respiratórias. A fragmentação do sono causa uma redução da resposta ventilatória à hipóxia e à hipercapnia. A disfunção neuromuscular das VAS também se acentua com fragmentação do sono. O intervalo de tempo até a resposta de despertar ao final do evento respiratório aumenta, e o número de pausas respiratórios por noite aumenta em consequiência da fragmentação do sono. Portanto, os despertares desencadeados pelas pausas respiratórias ocupam uma posição central no desenvolvimento de alterações cardiovasculares, déficits neuropsicológicos e reforço dos mecanismos de piora do distúrbio respiratório.

\section{Diagnóstico da SAOS}

Existe uma série de questionários padronizados e validados disponíveis na literatura médica para o diagnóstico qualitativo de SAOS. Contudo, o diagnóstico de confirmação é feito pela polissonografia. A polissonografia é o exame indicado e indispensável para o diag- 
nóstico quantitativo com índices que documentam o grau de gravidade da SAOS. Durante o polissonograma, o paciente é monitorizado com registro do eletroencefalograma, eletromiograma do queixo e das pernas, eletrooculograma (olhos), eletrocardiograma, fluxo de ar nasal e bucal, esforço respiratório e saturação de oxigênio. $O$ exame é realizado durante uma noite inteira, durando de 6 a 8 horas seguidas e todas as variáveis descritas acima são monitorizadas simultânea e continuamente. Apnéias/hipopnéias do sono são caracterizadas polissonograficamente como uma cessação da passagem de ar pelas vias aéreas superiores (VAS), com duração de pelo menos 10 segundos. Existem 4 tipos de pausas respiratórias:

apnéia central: há ausência de esforço respiratório e de fluxo de ar;

apnéia obstrutiva: persiste o esforço respiratório na ausência de passagem de ar pelas VAS;

apnéia mista: a pausa inicia como central e evolui para obstrutiva;

hipopnćias do sono são representadas por redução de pelo menos $50 \%$ na amplitude do fluxo aćreo com dessaturação de oxigêninio de no mínimo $4 \%$.

\section{Determinaçāo do nível de gravidade da SAOS}

A classificação do nível de gravidade da SAOS deve ser feito baseado nos índices polissonográficos, na intensi dade dos sintomas, impacto nas funções cognitivas, sociais e profissionais em função do sexo, idade c profissão c a presença de risco cardiovascular.

\section{SAOS leve:}

Associado a sonolência excessiva leve, dessaturação da oxihemoglobina discreta e baixo índice de apnéiahipopnéia entre 5 e 20 cventos por hora.

\section{SAOS moderada:}

Associado a sonolência excessiva moderada, dessaturação da oxi-hemoglobina moderada, moderado índice de apnéia-hipopnéia (entre 20 e 40 eventos/hora) e arritmias cardíacas.

\section{SAOS grave:}

Associado a sonolência excessiva intensa, dessaturação da oxihemoglobina grave, índice de apnéia-hipopnéia alto (acima de 40 eventos por hora) e arritmias cardíacas graves e sintomas de insuficiência cardíaca ou insuficiência coronariana.

\section{Tratamento}

A cadeia de eventos anormais que causam as pausas respiratórias na SAOS não é inteiramente conhecida. Os fatores que determinam o colapso das VAS durante o sono em pacientes com SAOS são diversos e relacionam-se com fatores anatômicos, fatores funcionais neuro-musculares das VAS e com alterações da neurofisiologia do controle respiratório do sistema nervoso central. Dada a incapacidade de se identificar qual fator está mais opcrante na SAOS, uma série de intervenções terapêuticas direcionadas especificamente aos diferentes pontos da cadeia de eventos anormais que causam as pausas respiratórias na SAOS passaram a serem propostas. Estas propostas de tratamento para SAOS levam em consideração os fatores predisponentes de alterações anatômicas das VAS $\mathrm{e}$ de alterações funcionais. Diminuir a colapsibilidade das VAS durante o sono, aumentar o tamanho das VAS, aumentar o tônus muscular das VAS c/ou rigidez das paredes das VAS e evitar fatores específicos que diminuam a contractilidade da musculatura das VAS formam a base do tratamento. O papel da musculatura dilatadora das VAS é claramente relacionado com o aumento do calibre das VAS e secundariamente com o aumento da rigidez das paredes $(41,43-45)$. A rigidez das paredes é modulada não somente pela atividade muscular mas também pelas estruturas ao redor das VAS, isto é, tecido conectivo, vasos sangüíneos e tecido adiposo. O tratamento da SAOS tem como objetivo implementar medidas que detenham o colabamento das VAS durante o sono. O tratamento está centrado em quatro pontos (Tabela 3): tratamento da obesidade, tratamento comportamental da SAOS (Tabela 4), tratamento físico e procedimentos cirúrgicos. O tratamento farmacológico da SAOS com um determinado agente invariavelmente não leva a resultados positivos em todo o universo de pacientes com apnéia. Porém, em pacientes selecionados, algumas drogas podem ser úteis no sentido de diminuir o

Tabela 3. Tratamento da Síndrome da Apnéia Obstrutiva do Sono.

Tratamento comportamental;

Tratamento da obesidade:

- não farmacológico:

farmacológico:

cirurgia bariátrica;

Uso de aparelhos:

- CPAP OU BiPAP.

- órteses intraorais removiveis;

Tratamento farmacológico da apnéia: em casos selecionados:

Tratamento cirúrgico: em casos selecionados, de acordo com a patologia identificada. 
Tabela 4. Recomendações e medidas comportamentais para pacientes com SAOS.

1) Perder peso;

2) Evitar álcool no mínimo 4 horas antes de dormir e antes das sonecas;

3) Evitar medicamentos sedativos tipo hipnóticos, antialérgicos, anti-histamínicos preferencialmente antes de dormir:

4) Evitar dormir de barriga para cima:

5) Evitar refeicões pesadas antes de dormir:

6) Evitar bebidas cafeinadas no mínimo 4 horas antes de dormir (café, chá, chocolate):

7) Evitar fumar no mínimo 4 horas antes de dormir;

8) Evitar comer no meio da noite:

9) Evitar fumar no meio da noite:

10) Evitar privação de sono:

11) Procurar dormir no mínimo 7,5 horas por noite:

12) Procurar manter horário relativamente constante para ir dormir e para acordar;

13) Levantar a cabeceira da cama cerca de 15 a 20 centímetros:

14) Eventualmente dormir sentado em uma poltrona;

15) Controlar infeções, inflamações, principalmente das vias aéreas.

número ou a duração dos períodos de apnéia. Mesmo que a SAOS não seja totalmente resolvida pelo tratamento farmacológico, pode haver melhora da aderência do paciente ao tratamento físico. O tratamento da obesidade deve ser enfatizado e definido de acordo com as necessidades individuais, preferencialmente envolvendo uma avaliação c intervenção multi-disciplinar c personalizada. Pacientes que segucm planos de alimentaçăo e atividade física individualizados são melhor sucedidos na perda de peso a longo prazo, quando comparados a pacientes que seguem cardápios prescritos pré-estabelecidos. A possibilidade de hipotiroidismo deve sempre ser descartada, uma vez que em alguns casos a reposição com tiroxina pode levar a desaparecimento da apnéia.
O tratamento farmacológico da obesidade está indicado $\mathrm{cm}$ pacientes com apnéia do sono, na presença de índice de massa corpórea maior que $25 \mathrm{~kg} / \mathrm{m}^{2}$ ou adiposidade central ou andróide, quando da falência do tratamento não farmacológico isoladamente. Pode-se, didaticamente, dividir os fármacos para o tratamento da obesidade em três grupos básicos: medicamentos anorexiantes de ação central, medicamentos termogênicos e medicamentos que afetam a absorção de nutrientes (Tabela 5), que serão discutidos detalhadamente em capítulo próprio. Teceremos aqui consideraçóes sobre particularidades do tratamento farmacológico da obesidade em pacientes com SAOS. As drogas termogênicas devem ser evitadas em pacientes obesos com apnéia do sono devido à estimulação simpática (efeito cronotrópico e promotor de elcvação da pressão arterial em indivíduos susceptíveis). No grupo de drogas que afetam a absorção de nutrientes, encontram-se os inibidores de lipases (orlistat), que atuam a nível gastrointestinal, diminuindo a absorção de gordura. Além de apresentar boa tolerabilidade, com perda de peso de cerca de $10 \%$ em 6 meses, apresenta a vantagem de não possuir efeito sistêmico, uma vez que os sinais e sintomas da SAOS nos sistemas nervoso e cardiovascular já são suficientemente proeminentes. Os medicamentos anorexiantes com ação simpatomimética levam a aumento do tônus noradrenérgico e/ou dopaminérgico. Os efeitos secundários são decorrentes do estímulo do sistema nervoso central (SNC) (insônia, irritabilidade, agitação psicomotora, sudorese) e dos efeitos cronotrópicos (notadamente taquicardia). Devido à incidência aumentada de doença cardiovascular e hipertensão arterial em indivíduos com SAOS, o uso de agentes anorexiantes catecolaminérgicos (dietilpropiona, fenproporex e mazindol) deve ser cvitado, devido aos

Tabela 5. Medicamentos disponiveis para tratamento da obesidade no Brasil.

\begin{tabular}{|c|c|c|}
\hline Classificação da droga & Mecanismo de ação & Medicamento \\
\hline Anorexígeno fenetilamínico & Agonista noradrenérgico & Dietilpropiona \\
\hline Anorexígeno fenetilamínico & Agonista noradrenérgico & Fenproporex \\
\hline Anorexígeno não fenetilamínico & Agonista noradrenérgico/dopaminérgico & Mazindol \\
\hline Sacietógeno fenetilamínico & Agonista serotoninérgico & Fenfluramina* \\
\hline Sacietógeno fenetilamínico & Agonista serotoninérgico & Dexfenfluramina* \\
\hline Antidepressivo & Inibidor da recaptação da serotonina & Fluoxetina \\
\hline Antidepressivo & Inibidor da recaptação da serotonina & Sertralina \\
\hline Sacietógeno não fenetilamínico & Agonista noradrenérgico/serotoninérgico & Sibutramina \\
\hline Termogểnico & Açõo simpatomimética & Efedrina \\
\hline Termogênico & Ação $\alpha_{2}$ antagonista & loimbina ${ }^{* *}$ \\
\hline Termogênico e anorexígeno & Ação $x$ z agonista central/simpatomimética & Fenilpropanolamina \\
\hline Malabsortiva & Inibidor de lipases & Orlistat \\
\hline
\end{tabular}

* Drogas retiradas do mercado em 1997.

* Eficácia experimental em estudos com animais. 
efeitos inotrópicos e ao risco de piora da hipertensão arterial. Esses agentes, porém, não são totalmente contra-indicados, podendo ser utilizados após avaliação clínica cuidadosa e na dependência da relação benefício: risco frente a cada caso, quando o tratamento clássico da obesidade com orientação nutricional e atividade física adaptada não tiver a aderência esperada. Os agentes farmacológicos de açào central com mecanismo serotoninérgico (fenfluramina e dexfenfluramina, e agentes anti-depressivos inibidores seletivos da recaptação da serotonina como a fluoxetina, a sertralina e a fluvoxamina) levam a aumento da saciedade. A fluoxetina e a sertralina são úteis $\mathrm{em}$ pacientes obesos com humor depressivo, no tratamento de pacientes com síndrome do comer compulsivo (do inglês binge eating syndrome) e em pacientes com bulimia nervosa. Em pacientes obesos com síndrome da apnéia obstrutiva do sono, a fluoxetina mostra-se também de grande utilidade, melhorando a ventilação e a capacidade respiratória, uma vez que suprime a fase REM do sono, onde ocorrem as maiores dessaturações de oxigênio. Os agentes scrotoninérgicos prolongam a meia-vida dos benzodiazepínicos, potencializam os efeitos do álcool e dos depressores do SNC, devendo por este motivo serem usados com cautela. Medicamentos anorexiantes de ação serotoninérgica (fenfluramina, dexfenfluramina e fluoxetina) podem ser utilizados, mas recomenda-se que os pacientes com apnéia moderada-grave estejam em uso de CPAP, já que eles podem acarretar aumento da sonolência. A documentação de casos de lesões valvulares cardíacas em alguns pacientes, que usaram por período prolongado a fenfluramina e a dexfenfluramina, levou à suspensão da comercialização destas drogas, embora não tenha sido possível apresentar uma relação causa-efeito. A sibutramina, um agente com mecanismo serotoninérgico e noradrenérgico, age através da inibição da recaptação de noradrenalina c serotonina, levando a redução de peso por diminuição da ingestão de alimentos (aumento da saciedade) e por aumento da atividade termogênica. Estudos com obesos normotensos mostraram um aumento discreto da freqüência cardíaca (da ordem de 4-6 bpm) e da pressão arterial (da ordem de $2-4 \mathrm{mmHg}$ ), o que justifica monitorização cautelosa destes parâmetros quando do seu uso em pacientes com SAOS. Não obstante, em um estudo com pacientes hipertensos, a perda média de $4,7 \mathrm{~kg} \mathrm{em}$ 12 semanas com sibutramina $10 \mathrm{mg}$ levou a reduções na PA diastólica e sistólica supina, respectivamente, da ordem de 4 e $5,5 \mathrm{mmHg}$. A redução de peso ocasiona aumento dos volumes pulmonares, redução dos tecidos faringeanos redundantes e da gordura retro- faríngea e mudanças no controle neuromuscular dos músculos da via aérea superior, levando a aumento da área faríngea e redução na colapsabilidade. A cirurgia bariátrica em centro especializado deve ser considerada em pacientes obesos mórbidos resistentes ao tratamento clínico. As medidas clínicas comportamentais incluem instruções para evitar álcool, fumo, sedativos, anti-histamínicos, privação de sono, decúbito dorsal (nesse sentido, em alguns pacientes que têm apnéias apenas na posição supina pode ser útil coser nas costas do pijama um bolso onde o paciente introduz uma bola de tênis para dormir) e refeições pesadas antes de dormir (vide tabela). Mudar o estilo de vida, procurando exercitar-se diariamente para desenvolver a musculatura e ajudar a perder peso, evitar o uso de antihistamínicos, evitar refeições pesadas especialmente no jantar e elevar a cabeceira da cama cerca de $15 \mathrm{~cm}$ (reduz a congestão vascular nos tecidos da cabeça e pescoço, melhorando a congestão nasal) podem reduzir a gravidade da SAOS em alguns pacientes (Tabela 4). Da mesma forma, condições como excursões a locais com muita altitude, insuficiência cardíaca congestiva e acidente vascular cerebral podem levar a piora da apnéia. Para casos mais graves de SAOS, a melhor alternativa é usar um sistema que compreende uma máscara ligada a um compressor mecânico, que bombeia ar sob pressão positiva nas vias aéreas superiores. Esses aparelhos que funcionam como "compressores de ar", sào chamados de CPAP (Continuous Positive Airway Pressure). Este aparelho cria uma pressão positiva que alarga as vias respiratórias promovendo bom resultados. As apnéias e os roncos desaparecem com o uso do aparelho e, a partir da primeira noite, ocorre melhora na função cognitiva, diminuição da sonolência diurna, da dessaturação de oxigênio e, quando presentes, das arritmias noturnas. Contudo, a aderência do paciente (60-70\% dos casos) ao uso continuado do aparelho é uma limitação importante já que, além do desconforto físico, alguns efeitos indesejáveis como claustrofobia, rinite e boca seca podem limitar o uso do aparelho.

Também foram desenvolvidos aparelhos intraorais removiveis para o tratamento de ronco e apnéia, com o objetivo de reposicionar anteriormente a língua e/ou a mandíbula. Entre os procedimentos cirúrgicos utilizados, a úvulo-pálato-faringoplastia (UPFP) é o mais comum, mas não é o tratamento de primeira escolha para SAOS por causa de resultados clínicos muito variados. Estudos polissonográficos pós-operatório da UPFP demonstram uma porcentagem de melhora de cerca de $50 \%$ e há poucos estudos de seguimento para determinação da eficácia da UPFP a longo prazo. Ideal- 
mente, pacientes menos obesos e com alterações específicas palatofaríngeas apresentam melhores chances de resposta cirúrgica. Complicações pós-operatórias abrangem rinolalia, refluxo nasofaríngeo, infecções c sangramento. Traqueostomia é mais eficiente, porém, com importante morbidade para o paciente, podendo ser uma medida temporária até correção da obesidade ou para casos graves com intolerância ao CPAP. Suspensão do hióide com avanço do genioglosso e avanço maxilo-mandibular são outras alternativas cirúrgicas. Medicações descongestionantes podem diminuir não apenas a congestão nasal, mas também exercer um papel nos pacientes que roncam, uma vez que também colaboram para a diminuição do edema faríngeo da SAOS. Estão em estudo, todavia sem indicação formal para o tratamento da apnéia antagonistas opióides, inibidores da enzima conversora da angiotensina, nicotina, buspirona, imipramina, trazodona.

\section{REFERÊNCIAS}

1. Hoffstein V. Is Snoring Dangerous to Your Health?. Sleep $1996 ; 19: 221-4$.

2. Guilleminault C, Stoohs R. Arousal, increased respiratory efforts, blood pressure and obstructive sleep apnea. J Sleep Res 1995;4(suppl 1):117-24.

3. He J, Kryeger MH, Zorick FJ, Conway W, Roth T. Mortality and apnea index in obstructive sleep apnea. Chest 1988:94:9-14.

4. Hillman DR. Sleep Apnea and Myocardial Infarction. Sleep 1993; 16:S23-24

5. Hla KM, Young TB, Bidwell T, Palta $M$, Skatrud JB, Dempsey J. Sleep apnea and hypertension: a population based study. Ann Intern Med 1994;120:382-8.

6. Hoffstein V, Mateika JH, Mateika S. Snoring and sleep architecture. Am Rev Resp Dis 1991:143:92-6.

7. Montplaisir J, Bêdard MA, Richer F, Rouleau. Neurobehavioral Manifestations in Obstructive Sleep Apnea Syndrome Before and After Treatment with Continuous Positive Airway Pressure. Sleep 1992; 15:S17-S19.

8. Partinen $M$, Guilleminault C. Daytime sleepiness and vascular morbidity at seven-year follow-up in obstructive sleep apnea patients. Chest 1990:97:27-32.

9. Gastaut H, Tassinari CA, Duron B. Étude polygraphique des manifestations, episodiques (hypniques et respiratoires), diurnes et nocturnes du syndrome de Pickwick. Revue Neurologie 1965;112:568-79.

10. Crampette L, Carlander B, Mondain M, Billiard M, Guerrier B, Dejean $Y$. Surgical alternatives to uvulopalatopharyngoplasty in sleep apnea syndrome. Sleep 1992; 15:S63-S68.

11. ICSD - International Classification of Sleep Disorders, revised: Diagnostic and Coding Manual: American Sleep Disorders Association, 1997.
12. Young T, Palta M, Dempsey J, Skatrud J, Webber S, Badr $\mathrm{S}$. The occurrence of sleep disordered breathing among middle aged adults. N Engl J Med 1993;328:1230-5.

13. Young T, Finn L, Hia M, Morgan B, Palta M. Snoring as Part of a Dose-Response Relationship Between Sleep-Disordered Breathing and Blood Pressure. Sleep 1996; 19(10):S202-206.

14. Young T. Sleep-Disordered Breathing in Older Adults: is it a Condition Distinct From That in Middle-Aged Adults? Sleep 1996: 19:529-30.

15. Aldrich M. Cardinal Manifestations of Sleep Disorders. In: Kryger, MH, Roth T, Dement WC. eds. Principles and Practice of Sleep Medicine $2^{\text {nd }}$ edition. Philadelphia: WB Saunders, 1994:413-25.

16. Kryger MH. Management of Obstructive Sleep Apnea. In: Kryger, MH, Roth T, Dement WC. eds. Principles and Practice of Sleep Medicine. $2^{\text {nd }}$ edition. Philadelphia: WB Saunders, 1994:584-90.

17. Philipson EA. Sleep apnea - a major public health problem. N Engl J Med 1993;328:1230-5.

18. Listro G, Stanescu DC, Veriter C, Rodenstein DO, AuberTulkens $G$. Patterns of Snoring in Sleep Apnea patients and Heavy Snorers. Sleep 1991:14(6):517-25.

19. Enright PL, Newman AB, Wahl PW, Manolio TA, Haponik EF, Boyle PJR. Prevalence and Correlates of Snoring and Apneas in 5,201 Older Adults. Sleep 1996;19:531-8.

20. Strollo PJ. Sanders MH. Significance and Treatment of Nonapneic Snoring. Sleep 1993: 16(5):403-8.

21. Manber R, Bootzin R, Acebo C, Carskadon M. The effects of Regularizing Sleep-Wake Schedules on Daytime Sleepiness. Sleep 1996:19(5):432-41.

22. Tavares S, Alóe F, Gentil V. Scaff M. Excessive Somnolence: Update Paper. São Paulo Med J / RPM 1996; $114(4) ; 1237-8$.

23. Hoddes E, Zarcone V, Smythe H, Phillips R. Dement WC. Quantification of sleepiness: a new approach. Psychophysiol 1973;10:431-6.

24. Johns MW. A New Method for Measuring Daytime Sleepiness: The Epworth Sleepiness Scale. Sleep $1991 ; 14: 540-5$.

25. Johns MW. Reliability and factor analysis of the Epworth Sleepiness Scale. Sleep 1992;15:376-81.

26. Akerstedt T. Gillberg M. Subjective and objective sleepiness in the active individual. Int J Neurosci 1990:52:29-37.

27. Casrkadon MA.- Guidelines for the Multiple Sleep Latency Test (MSLT): A Standard Measure of Sleepiness. Sleep 1986:9:519-24.

28. Carskadon MA, Brown ED, Dement WC. Sleep fragmentation in the elderly: Relationship to daytime sleep tendency. Neurobiol Aging 1981:23:321-7.

29. Bonnet MH. The effect of sleep disruption on performance, sleep and mood. Sleep 1985;8:1 1-9.

30. Bonnet $\mathrm{MH}$. Performance and sleepiness as a function of the frequency and placement of sleep disruption. Psychophysiol 1986;23:263-71. 
37. Stepanski E, Lamphere J, Roehrs T. Experimental sleep fragmentation in normal subjects. Int $\mathbf{J}$ Neurosci $1987: 33: 204-14$.

32. EEG arousals: scoring rules and examples. A preliminary report from the Sleep Disorders Atlas Task Force of the American Sleep Association. Sleep 1992:15:174-84

33. Bonnet MH. Sleep deprivation. In: Kryger, MH, Roth $\mathrm{T}$, Dement WC. eds. Principles and Practice of Sleep Medicine. $2^{\text {nd }}$ edition. Philadelphia: WB Saunders, 1994:50-68

34. Kimoff RJ. Sleep Fragmentation in Obstructive Sleep Apnea. Sleep 1996; 19(9):S61-S66

35. Chugh DK, Weaver TE, Dinges D. Neurobehavioral consequences of Arousals. Sleep 1996:19(10):S198-201.

36. Akersted T. Work hours, Sleepiness and Accidents. Introduction and Summary. J Sleep Res 1995;4(suppl 2): 1-3.

37. George CFP, Bordeau AC, Smiley A. Comparison of simulated driving performance in narcolepsy and sleep apnea patients. Sleep 1996;19:711-7.

38. Hoffstein V, Mateika S, Anderson D. Snoring: Is it in the ear of the beholder? Sleep 1994;17:422-526.

39. Issa FG, Sullivan CE. Upper airway closure pressures in obstructive sleep apnea. J Appl Physiol 1984:57:140-6.

40. Bradley T, Brown IG, Grossman RF, Zamel N, Martinez D, Phillipson EA, et al. Pharyngeal size in Snorers, nonsnorers, and patients with Obstructive Sleep Apnea. N Engl J Med 1986,315:1327-31.

41. Shepard JW, Gefter WB, Guilleminault C, Hoffman EA, Hoffstein V. Hydgel DW, et al. Evaluation of the Upper Airway in Patients with .Obstructive Sleep Apnea. Sleep $1991: 14: 361-71$.

42. Kuna ST, Sant'Ambrosio G. Pathophysiology of Upper Airway Closure During Sleep. JAMA 1991:22:1384-9.

43. Isono S, Remmers JE. Anatomy and physiology of upper airway obstruction. In: Kryger, MH, Roth T, Dement WC. eds. Principles and Practice of Sleep Medicine. $2^{\text {nd }}$ edition. Philadelphia: WB Saunders, 1994:525-36.

44. Hoffstein V. How and Why Should We Stabilize the Upper Airway. Sleep 1996: 19(9):S57-S60.

45. Schwab RJ. Properties of Tissues Surrounding the Upper Airway, Sleep 1996:19(10):S170-4

46. Schwartz AR, Gold AR, Schubert N, Sttryzak A, Wise RA, Permutt $S$, et al. Effect of weight loss on upper airway collapsibility in obstructive sleep apnea. Am Rev Respir Dis 1991;144:494-8.

47. Horner RL, Motor Control of the Pharyngeal Musculature and Implications for the Pathogenesis of Obstructive Sleep Apnea. Sleep 1996:19:827-53

48. Mezzanotte WS, Tangel DJ, White DP. Waking genioglossal EMG in sleep apnea patients versus normal controls (a neuromuscular compensatory mechanism). J Clin Invest 1992:89:1571-9.

49. Surratt PM, Mctier RF, Wilhoit SC. Upper airway muscle activation is augmented in patients with obstructive sleep apnea compared with that in normal subjects. Am Rev Resp Dis 1988; 137:889-94.
50. Petrof BJ, Hendricks JC, Pack Al. Does Upper airway Muscle Injury Trigger a Vicious Cycle in Obstructive Sleep Apnea? A Hypothesis. Sleep 1996; 19(6):465-71.

51. Carlson JT, Hedner J, Elam M, Ejnell H, Sellgren J, Wallin BG. Augmented resting sympathetic activity in awake patients with obstructive sleep apnea. Chest 1993:103:1763-8

52. Morgan BJ. Acute and chronic cardiovascular responses to sleep disordered breathing. Sleep 1996:19(10):\$2026-209

53. Ziegler MG, Nelesen R, Mills P, Ancoli-lsrael S, Kennedy B, Dimsdale JE. Sleep apnea, norepinephrine-release rate, and daytime hypertension. Sleep 1997:20;224-31.

54. Grustein RR. Metabolic aspects of sleep apnea. Sleep 1996: 19(10):S218-\$220

55. Veldhuis JD, Iranmanesh A. Physiological regulation of the human growth hormone (GH)-insulin-like growth factor type I (IGF-I) axis: Predominant impact of age, obesity, gonadal function, and sleep. Sleep 1996; 19(10):\$221S224.

56. Rangemark $C$, Hyedner JA, Carlson Gleerup $G$. Winther K. Platelet Function and Fibrinolytic Activity in Hypertensive and Normotensive Sleep Apnea patients. Sleep 1995: 18(3): 188-94

57. Horner RL. Autonomic consequences of arousal from sleep: mechanisms and implications. Sleep 1996: 19(10): 193-\$195

58. Partinen M, Gislason T. Basic Nordic Sleep Questionnaire (BNSQ): a quantitated measure of sleep complaints. J Sleep Res 1995:4, Suppl 1:150-5

59. Douglass AB, Bornstein R, Nino-Murgia $G$, et al. The sleep disorders questionnaire I: creation and multivariate structure of SDQ. Sleep 1994;17:160-7.

60. Issa FG. Sullivan CE. Alcohol, snoring and sleep apnea. J Neurol Neurosurg Psychiatry 1982;45:353-9.

61. Berthon-Jones M, Lawrence S, Sullivan C, Grustein R. Nasal continuous positive airway pressure: current realities and future. Sleep 1996;19:S131-135.

62. Krieger J, Kurtz D. Pettiau C, Sforza E, Trautman D. Longterm compliance with CPAP therapy in obstructive sleep apnea and in snorers. Sleep 1996;19:S131-135.

63. Schimidt-Nowara W, Lowe A, Wiegand L, Cartwright R, Guerra-Perez F, Menn $S$. Oral appliances for the treatment of obstructive sleep apnea. Sleep 1995; 18:501-10.

64. Sher A, Schecshtman KB, Piccirllo J. An American Sleep Disorders Association Review. The efficacy of surgical modifications of the upper airway in adults with obstructive sleep apnea. Sleep 1996; 19:156-7.

\section{Endereço para correspondência:}

Marcio C Mancini

Rua Bonsucesso 241

03305-000 São Paulo, SP

e-mail: marcio.mancini@attglobal.net 